\title{
Oral-Feeding Evaluation System for Preterm Infants
}

\author{
Venkata Ramadevi Nusum ${ }^{1}$, Mr. S. Sarath Chandra ${ }^{2}$, Mr. M Venkateswara Rao ${ }^{3}$ \\ Student of M. Tech, QISCET, Ongole, A.P, India ${ }^{1}$ \\ Associate Professor, Dept of ECE, QIS College of Engineering and Technology, Ongole, A.P, India ${ }^{2}$ \\ Associate Professor, Dept of BME, Osmania University, Hyderabad, Telangana, India ${ }^{3}$
}

\begin{abstract}
Feeding by sucking is the most important activity performed by babies on daily basis. Feeding and swallowing disorders are common in early infancy; early detection may cure certain fatal disorders in future. In Clinical experiments, improper feeding showed negative impacts on growth, neuro and cognitive developments, therefore it has become a high risk of neurodevelopmental delays in premature babies. In this study a method to assist infant's oral feeding and sucking behaviour is presented and experimentally validated. A monitoring system was developed to monitor the oral feeding events continuously and objectively. This device can be easily integrated in a commercially available feeding bottle, allowing clinical methods development for screening large numbers of subjects, in a quantitative manner. The results showed that the proposed approach showed accurate measurement of intra-oral pressure for neuromotor control analysis and estimation of milk volume delivered to the mouth.
\end{abstract}

Keywords: oral feeding disorder, infant monitoring system, oral feeding activities.

\section{INTRODUCTION}

Healthy, full-term infants are able to suck and swallow from birth. This enables them to take in all the nourishment they need from suckling at the breast or from feeding bottle. Oral feeding in infants needs to be efficient in order to preserve energy for growth. In addition, it should be safe so as to avoid aspiration, and it should not jeopardize respiratory status [1]. This is only possible if sucking, swallowing, and respiration are properly coordinated. Coordination means that the infant can suck efficiently and can swallow rapidly as the boluses are formed in the mouth in order to minimize the duration of airflow interruption. Oral feeding skills are defined as the infant's ability to organise and coordinate oral-motor functions efficiently so that it consumes enough calories for growth.

There are several circumstances that may compromise the normal for pre-terms suffering from broncho-pulmonary dysplasia (bpd), successful feeding can be hampered, on the one hand, by decreased oxygen saturation during feeding, deglutition apnea, and a higher respiratory rate (which is worse for pre-terms with bpd as the condition worsens). On the other hand, it may be hampered by the higher risk of neurological damage that leads to impaired sucking. The developmental course of sucking may be a predictor for neurological outcome later.

Congenital or acquired damage of the central nervous system may lead to feeding problems in the neonatal period such as slow or weak sucking. This could be the first indication that the infant has neurological problems. Dysphagia is common in infants suffering from cerebral palsy or other neurological developmental disorders.
The reasons for studying the preconditions for sucking and how an infant sucks are to determine the infant's readiness to feed orally and to detect the nature of its feeding problems. In addition, an abnormal sucking pattern may be an indication that the infant's neurological development is not progressing normally. We used the Early Feeding Skills Assessment to determine whether an infant was ready to feed orally [2]. This observational scale is used to monitor the infant before, during and after each feeding. In general, to assess the way infants suck, a distinction is made between clinical feeding assessment and swallowing assessment. Seven other diagnostic tools have been described in the literature: four are designed for breastfeeding only, two for bottle-feeding only, and one is applicable to both feeding situations. The reliability and user-friendliness of these tools are fair to poor.

To date, we lack a user-friendly, reliable, and non-invasive tool that can be used for both breastfeeding and bottlefeeding and that objectively measures the coordination between sucking, swallowing and breathing, and sucking and swallowing movements. On the one hand, such a diagnostic tool would be useful to determine what kinds of interventions are required to facilitate sucking and swallowing. On the other hand, it would be useful if it could make some predictions regarding the future development of the infant.

\section{METHODOLOGY}

During NS, fluid moves primarily due to change in pressure. With the oral cavity sealed, as the jaw and tongue drop down, the cavity is enlarged. This 
Vol. 4, Issue 1, January 2017

enlargement creates negative intra-oral pressure, suction, which draws fluid into the mouth and propels the expressed fluid backwards toward the pharynx for the swallow. Jaw and tongue movements are also involved in the propulsion of fluid. As the tongue compresses the nipple, sufficient positive pressure, compression, is created by the jaw and the front part of the tongue pressing the nipple against the hard palate to draw the fluid from the nipple. The tongue plays a key role in all aspects of sucking by helping to seal the oral cavity. It does so anterior, in conjunction with the lower lip, and posterior, by sealing against the soft palate during swallowing. In addition, the tongue stabilises the lower jaw and transports the bolus to the pharynx. The jaw provides a stable base for movements of the tongue, lips and cheeks.

The proposed system contains a sucking pressure sensing device. The sucking pressure sensing device was placed in the infant's mouth to obtain the sucking pressure.

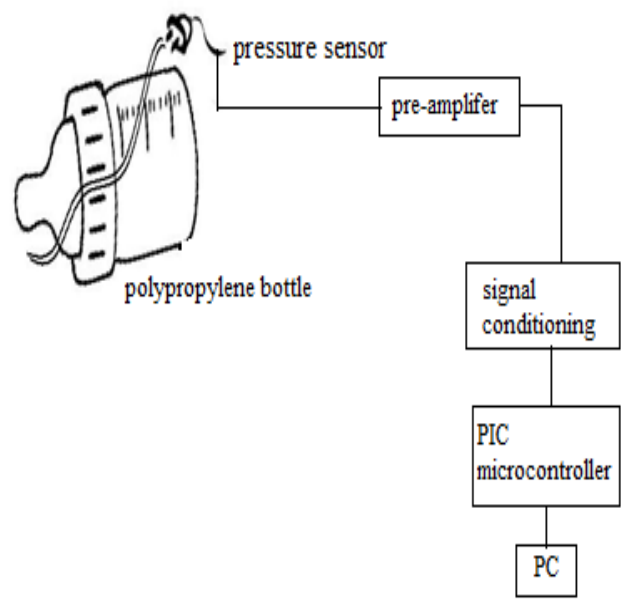

Fig1 Sucking pressure sensing device

Fig. 1 shows the design of the sucking pressure sensing device. The pressure sensor which contains two pressure inputs P1 (+) and P2 (-), was used in this device. The device accesses the changes in sucking pressure between $\mathrm{P} 1$ and $\mathrm{P} 2$, and then transmits this raw data to the data acquisition module. A transparent rubber tube is used to connect the pressure sensor and the bottle to measure the pressure difference between the general atmospheric pressure and the inner pressure of the polypropylene bottle. Finally, the pressure sensor will convert a measured pressure into an electrical output signal.

The data acquisition module mainly contains the following parts: front-end pre-amplifier and signal conditioning circuits, a microcontroller with an analog-to-digital converter (ADC). The microcontroller considered is PIC 16f877, which has got a 10-bit ADC. The microcontroller is programmed to acquire the analog pressure signal at 200 samples/ sec. These signals are transferred to PC using USB connectivity. In PC a MATlab programme is developed to identify the number of sucks per swallow, peak sucking pressure, volume of milk per suck.

\section{RESULTS AND DISCUSSION}

Fig. 3 shows a typical 10 seconds pressure burst: experimental data confirm that intra-oral pressure is in the range of $[-140+15] \mathrm{mmHg}$. This pressure waveform and the values are for normal group, whereas these values are changing quite erratically for a premature baby. This is the preliminary study, and the study can be further extended by considering statistically significant number of cases for different classes.

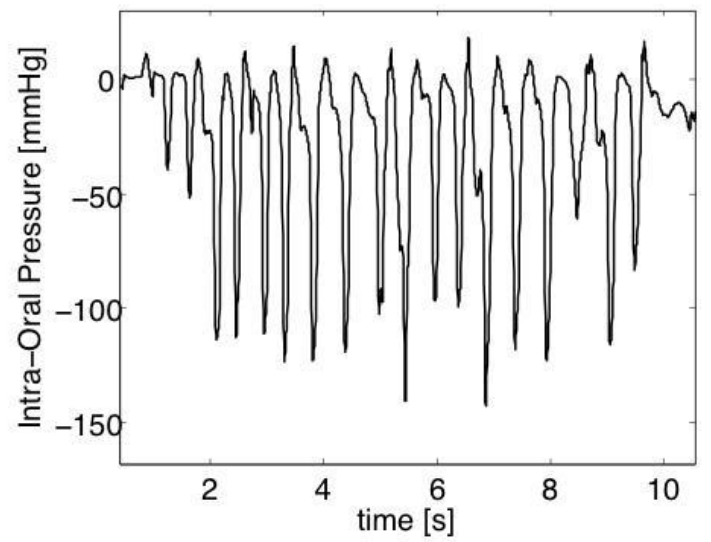

Fig 2 Intra-oral pressure of a 1 week newborn recorded during a feeding task.

\section{REFERENCES}

[1] Fabrizio Taffoni et.al. "Ecological Sucking Monitoring of Newborns", IEEE SENSORS JOURNAL, VOL. 13, NO. 11, NOVEMBER 20134561

[2] Eleonora Tamilia et.al. "An Automated System for the Analysis of Newborns' Oral-Motor Behavior" IEEE TRANSACTIONS ON NEURAL SYSTEMS AND REHABILITATION ENGINEERING, VOL. 24, NO. 12, DECEMBER 2016 